\title{
Relación de la variación del almacenamiento de agua local y el gravímetro superconductor en el Observatorio Geodésico TIGO, Concepción, Chile
}

\section{Relationship of local water storage variations and superconducting gravimeter data at the} Geodetic Observatory TIGO, Concepción, Chile

Fecha de entrega: 6 de septiembre 2012 Fecha de aceptación: 10 de diciembre 2012

\section{Ghislaine Rossel ${ }^{1}$, Andreas Güntner ${ }^{2}$, Benjamin Creutzfeldt ${ }^{2}$, Hartmut Wziontek $^{3}$, Thomas Klügel ${ }^{3}$, Pedro Tume ${ }^{4}$, Mauricio Villagrán ${ }^{5}$, Theresa Blume $^{2}$ y Hayo Hase ${ }^{3,6}$}

${ }^{1}$ Ingeniería Operacional y Gestión de Redes, ESSBIO, Pedro Aguirre Cerda 1129, Piso 2, Concepción, Chile, ghislaine.rossel@essbio.cl

2 Helmholtz-Centre Potsdam - German Research Centre for Geosciences GFZ, Telegrafenberg14473 Potsdam, Germany, guentner@gfz-potsdam.de, benjamin.creutzfeldt@gfz-potsdam.de,blume@gfz-potsdam.de

${ }^{3}$ Federal Agency for Cartography and Geodesy BKG, Richard-Strauss-Allee 11 - 60598 Frankfurt Main, Germany, hartmut.wziontek@bkg.bund.de,kluegel@fs.wetzell.de

${ }^{4}$ Departamento de Ingeniería Civil, Universidad Católica de la Santísima Concepción, Concepción, Chile, ptume@ucsc.cl

5 Departamento de Ingeniería Hidráulica y Ambiental, P. Universidad Católica de Chile, Vicuña Mackenna 4860, Santiago, Chile, mvillagran@ing.puc.cl

${ }^{6}$ Observatorio Geodésico TIGO, Camino Einstein km 2.5, Universidad de Concepción, Casilla 4036, Correo 3 Concepción, Chile, hayo.hase@tigo.cl

Los gravímetros superconductores SGs miden variaciones temporales del campo de gravedad de la Tierra con alta precisión, y han sido usados tradicionalmente en aplicaciones geodésicas. Recientes estudios han mostrado que el componente de disturbio de la señal en las series de tiempo del SG puede ser usado inversamente como un sensor hidrológico-sensible a cambios en las masas de agua a su alrededor. Los SGs proveen mediciones únicas de variaciones totales de almacenamientos de agua, suma de la variación en almacenamientos de nieve, suelo no saturado y agua subterránea, para escalas locales de varios cientos de metros de distancia. En este estudio se investigó la relación entre la hidrología local y la gravedad para el sensor SG ubicado en el Observatorio Geodésico Integrado Transportable TIGO en Concepción, Chile. Las series de tiempo del SG son comparadas con la respuesta gravimétrica calculada por un modelo geodésico usando mediciones de humedad del suelo hasta $2.6 \mathrm{~m}$ de profundidad y un Modelo de Elevación Digital para un radio de $2 \mathrm{~km}$ alrededor del SG. Los resultados muestran que la variación de humedad y la respuesta gravimétrica están relacionadas con la topografia y profundidad de análisis. La variación estacional de la gravedad es de hasta $12 \mu \mathrm{Gal}$, indicando que la importancia de la variación de los almacenamientos de agua está en la profundidad de la zona no saturada y en las aguas subterráneas.

Palabras clave: gravímetro superconductor, señal de disturbio, almacenamiento de agua, respuesta gravimétrica
Superconducting gravimeters SGs measure temporal variations of the Earth's gravity field with high precision. SGs have traditionally been used in geodetic applications. Recent studies, however, have shown that the disturbing signal component in SG time series can inversely be used as a hydrological sensor. Being sensitive to water mass changes in their surroundings, SGs provide unique measurements of total water storage variations, sum of storage variations in the snow cover, the unsaturated soil, and the groundwater, at local scales of several hundreds of meters. In this study, the relationship between local hydrology and gravity for the SG located at the Geodetic Observatory TIGO in Concepción, is investigated. SG time series are compared to the gravimetric response calculated by a geodetic model using soil moisture measurements to a depth of $2.6 \mathrm{~m}$ and a Digital Elevation Model for an area of $2 \mathrm{~km}$ around the $S G$. The results show that variations in moisture and gravimetric response are related to the topography and depth of analysis. Seasonal gravity variations of up to $12 \mu \mathrm{Gal}$ give indication of important water storage variations, which are related to the depth of unsaturated zones and groundwater.

Keywords: superconducting gravimeter, disturbing signal, water storage, gravimetric response 


\section{Introducción}

La relación entre la hidrología y la gravedad está atrayendo cada vez más la atención de las ciencias hidrológicas y geodésicas. Desde una perspectiva hidrológica la estimación del almacenamiento de agua y la variación espacio-temporal es importante porque permite cuantificar balances de agua para el uso y manejo efectivo del recurso. Las mediciones directas de las variaciones de los almacenamientos de agua son una tarea difícil, ya que se realizan en puntos específicos y están asociadas a un alto nivel de incertidumbre. Por lo que las observaciones de gravedad se están convirtiendo en una promisoria herramienta ya que la permanente observación gravimétrica podría llegar a proveer de gran información sobre el cambio de estos sistemas. Desde la perspectiva geodésica el efecto de la gravedad hidrológica local interfiere en la señales adhiriendo alteraciones a las mediciones gravimétricas debido a que la precisión de las mediciones de gravedad in situ se ven afectadas por las variaciones de almacenamientos de agua, ya sean las aguas subterráneas, humedad del suelo, agua almacenada en la superficie, etc., las cuales deben ser eliminadas desde los registros gravimétricos.

Según Creutzfeldt et al. (2008), Bonatz en el año 1967 fue el primero en estudiar la relación entre hidrología y gravedad a través de la simulación del efecto de la humedad del suelo sobre las mediciones de gravedad, concluyendo que no era útil para la aplicación geodésica, ya que era necesario el desarrollo de gravímetros de alta precisión porque la humedad del suelo puede contribuir a la señal gravimétrica en el rango de $10 \mathrm{mGal}$. Sin embargo, más adelante fueron desarrollados gravímetros de mayor precisión como por ejemplo el gravímetro superconductor SG. Estos gravímetros relativos son de alta precisión con mediciones de resolución de $0.01 \mu \mathrm{Gal}$, pero debido a alteraciones atmosféricas o de origen sísmico su precisión disminuye a rangos entre 0.1 a 1 $\mu \mathrm{Gal}$ (Creutzfeldt et al., 2008).

Para trasladar los cambios de los almacenamientos de agua a cambios en la gravedad, actualmente se han implementado variados modelos dependiendo del sistema en estudio. Generalmente se usan prismas rectangulares para la obtención de la topografía y el subsuelo (Hasan et al., 2006). También existen otros modelos usados como segmentos esféricos o polígonos 3D TINs. Estos modelos permiten el cálculo exacto de las variaciones gravimétricas producto de las variaciones en los almacenamientos de agua considerando la topografía y la distribución espacial de las masas (Creutzfeldt et al., 2008). Intentando hacer más eficiente los métodos en relación al tiempo de procesamiento y la precisión, Leirião (2007) propone el uso de diferentes modelos para diferentes aéreas de influencia alrededor del gravímetro. Para el área más cercana alrededor del gravímetro usa la ecuación del prisma que integra completamente cada volumen de elemento, para reducir el tiempo computacional usa la ecuación de punto masa para distancias largas y para distancias intermedias usa la ecuación de MacMillan.

Para investigar cómo influye la topografía y la precisión de los modelos de elevación digital DEM en el cálculo de la gravedad, cómo influyen los diferentes componentes de almacenamiento en la señal gravitacional y cómo varía el radio de influencia para cada componente, Creutzfeldt et al. (2008) aplica un modelo 4D usando como ejemplo el SG del Geodetic Observatory Wettzell en Alemania. Se evalúa la posible máxima influencia de las variaciones de agua de todos los componentes hidrológicos y se analiza la influencia real de los datos hidrológicos sobre las mediciones del SG, demostrando que entre el $52 \%$ y el $80 \%$ de la señal hidrológica local de la gravedad es generada en un radio de $50 \mathrm{~m}$ alrededor del gravímetro superconductor SG. Aproximadamente el $90 \%$ de la señal es generada en un radio de influencia de $1000 \mathrm{~m}$ alrededor del gravímetro SG.

Otros estudios se han enfocado en la combinación de modelos hidrológicos espaciales semi-distribuidos y observaciones de gravedad. Hasan et al. (2008) calibra conjuntamente un modelo de humedad del suelo y un modelo de almacenamiento en pendiente de Boussinesq, para la escorrentía de aguas subterráneas y la señal de gravedad in situ, respectivamente. Creutzfeldt et al. (2010), centran su atención en un pequeño radio de influencia cercano al gravímetro donde las variables hidrológicas y su efecto sobre la gravedad son más pronunciadas en la profundidad que en el área. Debido a esto resuelven las variables de profundidad en un enfoque 1D centrándose separadamente en cada componente de almacenamiento y calculan la respuesta gravimétrica de la variación del almacenamiento y las comparan con los datos del SG. 
Basándose en este estudio se investiga la relación entre la hidrología y la gravedad, donde se evalúa la influencia de la variación del contenido de humedad del suelo en las mediciones gravimétricas del Observatorio Geodésico Integrado Transportable TIGO.

\section{Datos y metodología}

Los datos para este estudio fueron obtenidos por el Observatorio TIGO ubicado sobre un cerro, en Camino

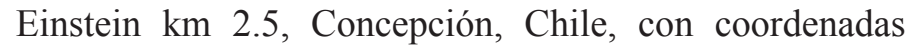
geográficas $36^{\circ} 50.568^{\prime} \mathrm{S}, 73^{\circ} 1.513^{\prime} \mathrm{O}$, y altura $160 \mathrm{~m}$ sobre el nivel medio del mar (ver Figura 1). TIGO está gestionado por el BKG (German Federal Agency for Cartography and Geodesy) en colaboración con la Universidad de Concepción. La zona en estudio se caracteriza por tener precipitaciones medias anuales de $1500 \mathrm{~mm}$, presentar una evapotranspiración potencial promedio anual de $1420 \mathrm{~mm}$ y una temperatura media anual de $12^{\circ} \mathrm{C}$. Los parámetros climáticos de relevancia hidrológica, temperatura del aire, humedad relativa, velocidad del viento, precipitación, presión atmosférica, son medidos por TIGO, y el período de estudio corresponde a enero del año 2006 hasta enero 2011.

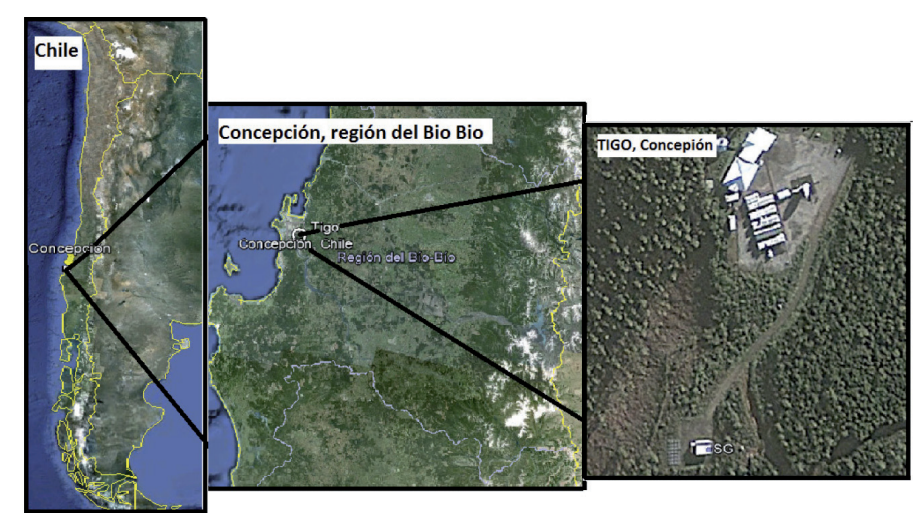

Figura 1: Ubicación observatorio TIGO, imagen satelital obtenida de Google Earth

El área alrededor de la estación donde se encuentra emplazado el gravímetro es rural, predominando bosques, con una topografía accidentada. El suelo es caracterizado a través de muestras a diferentes profundidades, las cuales se dividieron en sub muestras para determinar así la humedad y la granulometría, esta última se obtiene por medio de tamizaje húmedo, para separar las arenas, y por el método de pipeta, para limos y arcillas.
Luego por medio del triángulo textural USDA (Davis y Bennett, 1927), se procedió a la clasificación del suelo en el Laboratorio de Sedimentología del Departamento de Ciencias de la Tierra, Universidad de Concepción (Tabla 1).

Tabla 1: Clasificación del suelo

\begin{tabular}{|l|l|c|c|c|}
\hline Parámetros & unidad & Muestra 1 & Muestra 2 & Muestra 3 \\
\hline Profundidad & $\mathrm{m}$ & 0.6 & 0.9 & 1.2 \\
\hline Arena & $\%$ & 22 & 25 & 23 \\
\hline Limo & $\%$ & 54 & 49 & 63 \\
\hline Arcilla & $\%$ & 24 & 26 & 14 \\
\hline Descripción & $\begin{array}{c}\text { Franco } \\
\text { limosa }\end{array}$ & Franco & $\begin{array}{c}\text { Franco } \\
\text { limosa }\end{array}$ \\
\hline
\end{tabular}

Basándonos en los resultados de la Tabla 1, es posible clasificar el suelo en el área circundante al gravímetro SG38, como suelo Franco limoso a los 0.6 m, Franco a los $0.9 \mathrm{~m}$ y Franco limoso a los $1.2 \mathrm{~m}$. La humedad del suelo fue determinada por 5 sensores del tipo sondas TDR (Time Domain Reflectometry) Tipo Trime-EZ instalados por BKG a $0.15,0.40,0.8,1.4$ y $2.2 \mathrm{~m}$ de profundidad, ubicados a $3 \mathrm{~m}$ desde la estructura donde se encuentra ubicado el gravímetro. Tomando como base los resultados de la clasificación del suelo y la ubicación de los sensores, el suelo en su profundidad se divide en distintas zonas, de $0.0-0.2 \mathrm{~m}, 0.2-0.6 \mathrm{~m}, 0.6-1.0 \mathrm{~m}, 1.0-1.8 \mathrm{~m}$ y $1.8-2.6 \mathrm{~m}$, asumiendo representativo una de las sondas por cada zona.

\section{Humedad del suelo}

Los datos de humedad del suelo son registrados cada 20 min, cada serie de tiempo fue inspeccionada visualmente a través de gráficos, quitando de esta manera los errores e incertidumbres. Dos sensores presentaron errores en la toma de sus datos a partir del término del primer año. Es por ello que estos datos fueron reemplazados para los años posteriores con datos de los sensores con mayor correlación en el período de medida común. A continuación la Figura 2 muestra las variaciones temporales de humedad del suelo en cada sensor, los períodos de mayor humedad corresponden a los meses de invierno, los que a su vez son los más lluviosos. La mayor variación, es decir, la diferencia entre la menor y mayor humedad registrada, se presenta en el primer sensor a $0.15 \mathrm{~m}$, esto se debe a que la lluvia caída al suelo filtra más rápidamente a las capas superiores. 


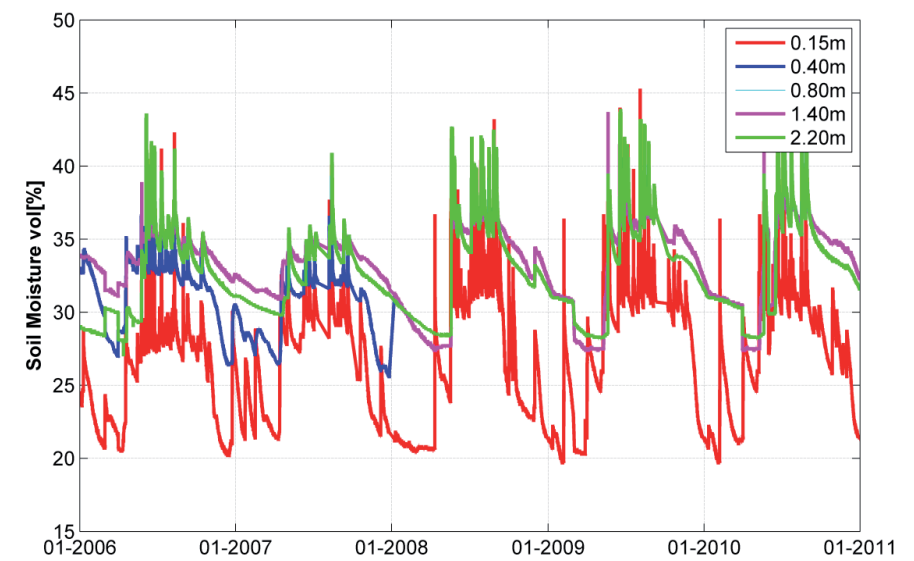

Figura 2: Variación de la humedad en el suelo de cada sensor, la línea roja corresponde al sensor a $0.15 \mathrm{~m}$, la línea azul al sensor a $0.4 \mathrm{~m}$, la línea celeste al sensor a $0.6 \mathrm{~m}$, la línea magenta al sensor a $1.4 \mathrm{~m}$ y la línea verde al sensor a $2.2 \mathrm{~m}$.

\section{Gravímetro}

Los gravímetros son instrumentos que realizan mediciones correspondientes a la variación espacial de la fuerza de gravedad, sin embargo, existen gravímetros de alta resolución y estabilidad, que son capaces de medir variaciones temporales de esta fuerza. En este estudio los datos de variación temporal de la fuerza de gravedad son obtenidos por el gravímetro superconductor SG38 en combinación con el gravímetro absoluto FG5-227. El gravímetro se encuentra ubicado sobre una estructura que tiene $6.2 \times 3.5$ $\mathrm{m}$ (largo x ancho) y está $0.25 \mathrm{~m}$ sobre el nivel del suelo. Los datos se encuentran disponibles a partir de enero del año 2006 hasta enero del 2011 y fueron recogidos en intervalos de una hora (TIGO 2011). En la Figura 3 se presentan los valores de gravedad residual, los cuales corresponden a los datos gravimétricos del SG, corregidos por los efectos no hidrológicos, por ejemplo mareas terrestres, mareas oceánicas y cambios de la masa atmosférica. Para el periodo 07/2008-12/2009, debido a la refacción del SG, la serie de tiempo de gravedad residual fue reconstruida por mediciones semanales de un gravímetro absoluto (FG5-227) efectuado en el mismo lugar.

\section{Respuesta gravimétrica}

La respuesta gravimétrica de la variación de humedad en el suelo (zona no saturada) será determinada mediante la ecuación de MacMillan (Leirião, 2007; Creutzfeldt et al. 2008).

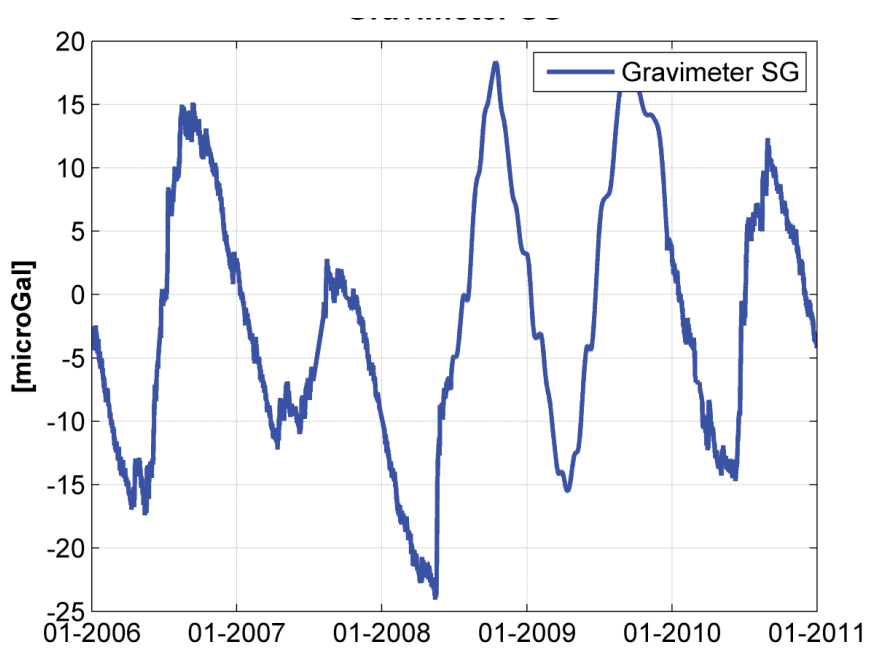

Figura 3: Serie de tiempo, variación de las mediciones gravimétricas, gravímetro SG38

El modelo para simular la respuesta gravimétrica es codificado en MATLAB, basado en la ecuación de MacMillan, donde la componente $z$ del cambio de gravedad, debido a la homogeneidad de las variaciones de almacenamientos de agua, es calculada para cada cuerpo y el efecto total de la gravedad se obtiene de la suma de los cambios de gravedad en cada cuerpo elemental.

$\Delta \mathrm{g}=\mathrm{G} \Delta \rho \Delta \mathrm{x} \Delta \mathrm{y} \Delta \mathrm{z}\left[-\frac{\mathrm{z}}{\mathrm{d}^{3}}-\frac{5}{24} \frac{\left(\alpha \mathrm{x}^{2}+\beta \mathrm{y}^{2}+\omega \mathrm{z}^{2}\right) \mathrm{z}}{\mathrm{d}^{7}}+\frac{1}{12} \frac{\omega \mathrm{z}}{\mathrm{d}^{5}}\right]$

Con $\alpha=2 \Delta x^{2}-\Delta y^{2}-\Delta z^{2}, \beta=-\Delta x^{2}+2 \Delta y^{2}-\Delta z^{2}, \omega=$ $-\Delta x^{2}-\Delta y^{2}+2 \Delta z^{2}, \mathrm{y} d=\sqrt{x^{2}+y^{2}+z^{2}}$, donde $(x, y, z)$ es el centro de las coordenadas de un cuerpo elemental, relativo al sensor (m), $\Delta x, \Delta y, \Delta z$, son las longitudes de los lados de la celda $\Delta x y=\Delta x=\Delta y(\mathrm{~m}), G$ es la constante de gravitación universal $\left(6.673 \cdot 10^{-11} \mathrm{Nm}^{2} / \mathrm{kg}^{2}\right)$ y $\Delta \rho$ es el cambio de densidad.

El efecto de la variación de la humedad en el suelo es calculado asumiendo una variación homogénea en el cambio de densidad, aunque la variación de humedad en el suelo respecto a la profundidad y superficie son equivalentes a cambios en el nivel de agua. El cambio de densidad no se distribuye en forma heterogénea en cada cuerpo; sin embargo, este error de simplificación se puede minimizar reduciendo el espesor de las líneas en el modelo.

Para reducir el error de aproximación de la ecuación de MacMillan en el área cercana al gravímetro, se realiza una discretización anidada en el dominio del modelo 
presentada por Creutzfeldt et al. (2008), la cual permite una mayor resolución en la zona próxima al centro del DEM; la resolución de 0.25 m, 2.5 m y 10 m de los DEM variará con el radio del dominio para $\mathrm{R}=50 \mathrm{~m}, \mathrm{R}=500$ $\mathrm{m}$ y $\mathrm{R}=2000 \mathrm{~m}$, respectivamente. Esto se realiza para el área de un cuadrado de lado $4 \mathrm{~km}$, con el gravímetro SG38 ubicado en el centro. El uso de un DEM es necesario para distribuir las variaciones de humedad del suelo en la topografía. La Figura 4 muestra la extensión espacial total de la discretización anidada del dominio del modelo, donde $\mathrm{R}$ corresponde a la mitad de la longitud del lado del cuadrado del dominio y $\Delta x y$ es el tamaño de la celda que varía con el dominio $\mathrm{R}$, las zonas verdes corresponden a las mayores alturas y las zonas café a las menores altura, el gravímetro SG38 se ubica al centro.

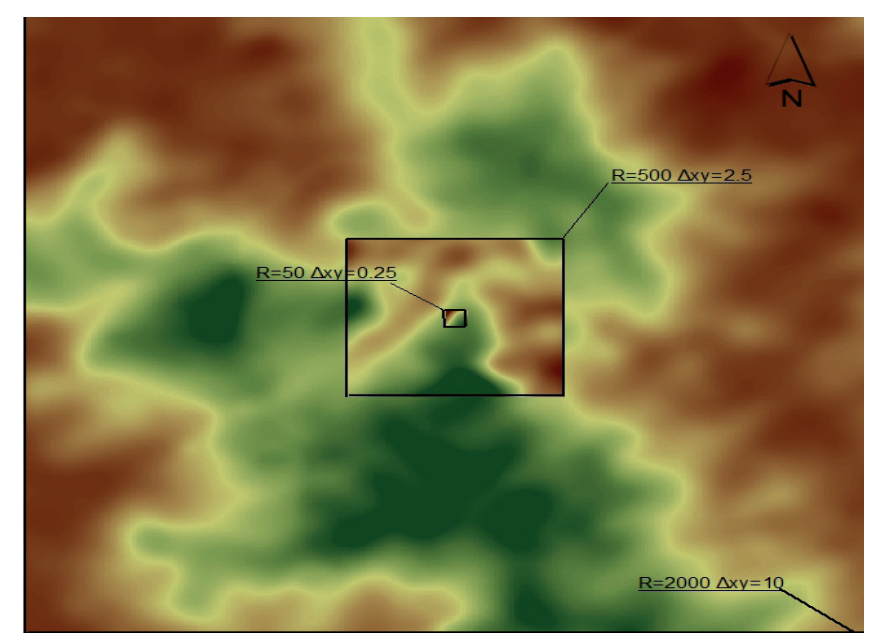

Figura 4: Extensión espacial total de la discretización anidada del dominio del modelo.

La influencia de las masas hidrológicas sobre el sensor gravimétrico será estimada asumiendo un máximo cambio hipotético basado en condiciones de borde reales. En este caso se considera el efecto de la estructura donde se encuentra el gravímetro, se supondrá que no existe infiltración bajo ella. Las respuestas gravimétricas obtenidas de la humedad del suelo serán comparadas con las mediciones obtenidas por el gravímetro SG38.

\section{Resultados}

Los datos de humedad del suelo están disponibles hasta los $2.6 \mathrm{~m}$ de profundidad; sin embargo, la zona de almacenamiento de humedad alcanza mayores profundidades, por lo mismo se realiza el análisis, también, suponiendo que la humedad del sensor presente a los $2.2 \mathrm{~m}$, es constante hasta los $20 \mathrm{~m}$. Los espesores de capa del suelo son de $0.1 \mathrm{~m}$ hasta los $2.6 \mathrm{~m}$, y desde ahí se consideran líneas de espesor de $1 \mathrm{~m}$ hasta los $20 \mathrm{~m}$. En la Tabla 2 se muestran los resultados asumiendo un cambio de densidad de $1000 \mathrm{~kg} / \mathrm{m}^{3}$ en cada horizonte.

Tabla 2: Respuesta gravimétrica en $\mu \mathrm{Gal}$, a diferentes radios $\mathrm{R}$ y diferentes profundidades

\begin{tabular}{|c|c|c|c|c|}
\hline \multirow{2}{*}{ Horizonte } & \multicolumn{4}{|c|}{ Respuesta $10^{-2} \mathrm{~m} / \mathrm{s}^{2}$} \\
\cline { 2 - 5 } & $\mathrm{R}=50 \mathrm{~m}$ & $\mathrm{R}=500 \mathrm{~m}$ & $\mathrm{R}=2000 \mathrm{~m}$ & Resp.grav.total \\
\hline $0.0-0.2 \mathrm{~m}$ & 1.0944 & 0.9607 & 0.1786 & 2.2337 \\
\hline $0.2-0.6 \mathrm{~m}$ & 1.7729 & 0.9845 & 0.1807 & 2.9381 \\
\hline $0.6-1.0 \mathrm{~m}$ & 2.2814 & 1.0082 & 0.1829 & 3.4725 \\
\hline $1.0-2.6 \mathrm{~m}$ & 3.2369 & 1.1018 & 0.1916 & 4.5363 \\
\hline $2.6-10 \mathrm{~m}$ & 33.9774 & 14.8091 & 2.2944 & 51.0809 \\
\hline $10-20 \mathrm{~m}$ & 26.3847 & 19.3519 & 2.8363 & 48.5729 \\
\hline
\end{tabular}

La respuesta gravimétrica es mayor en el área cercana al gravímetro $\mathrm{R}=50 \mathrm{~m}$, luego disminuye a medida que aumenta el radio $\mathrm{R}$, es por ello que esta área es la de mayor influencia en la respuesta total.

La distribución espacial de la variación gravimétrica se muestra en la Figura 5 para el área completa, al centro se ubica la estructura donde se encuentra el gravímetro y la variación de masas es 0 , los valores negativos corresponden a las zonas rojas, las que están asociadas a los puntos de mayor altura, es decir, sobre el gravímetro SG38.

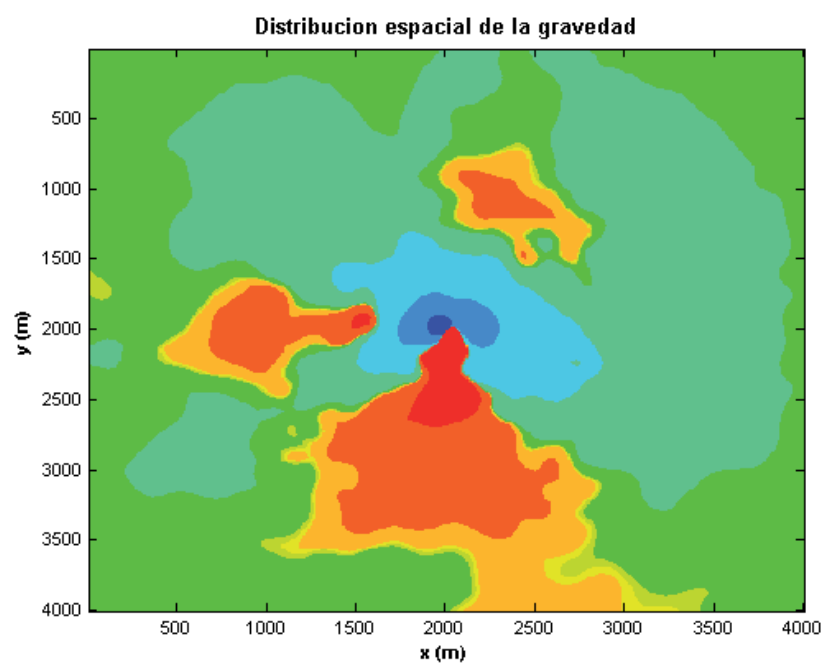

Figura 5: Distribución espacial de la variación gravimétrica para $\mathrm{R}=2000 \mathrm{~m}$ 
La distribución espacial de la variación gravimétrica para los diferentes radios con su respectiva resolución, se muestra en las Figura 6, donde el cuadro amarillo ubicado en el centro de $\mathrm{R}=50 \mathrm{~m}$, corresponde a la casa gravimétrica donde está ubicado el SG y la variación de masas es 0 .

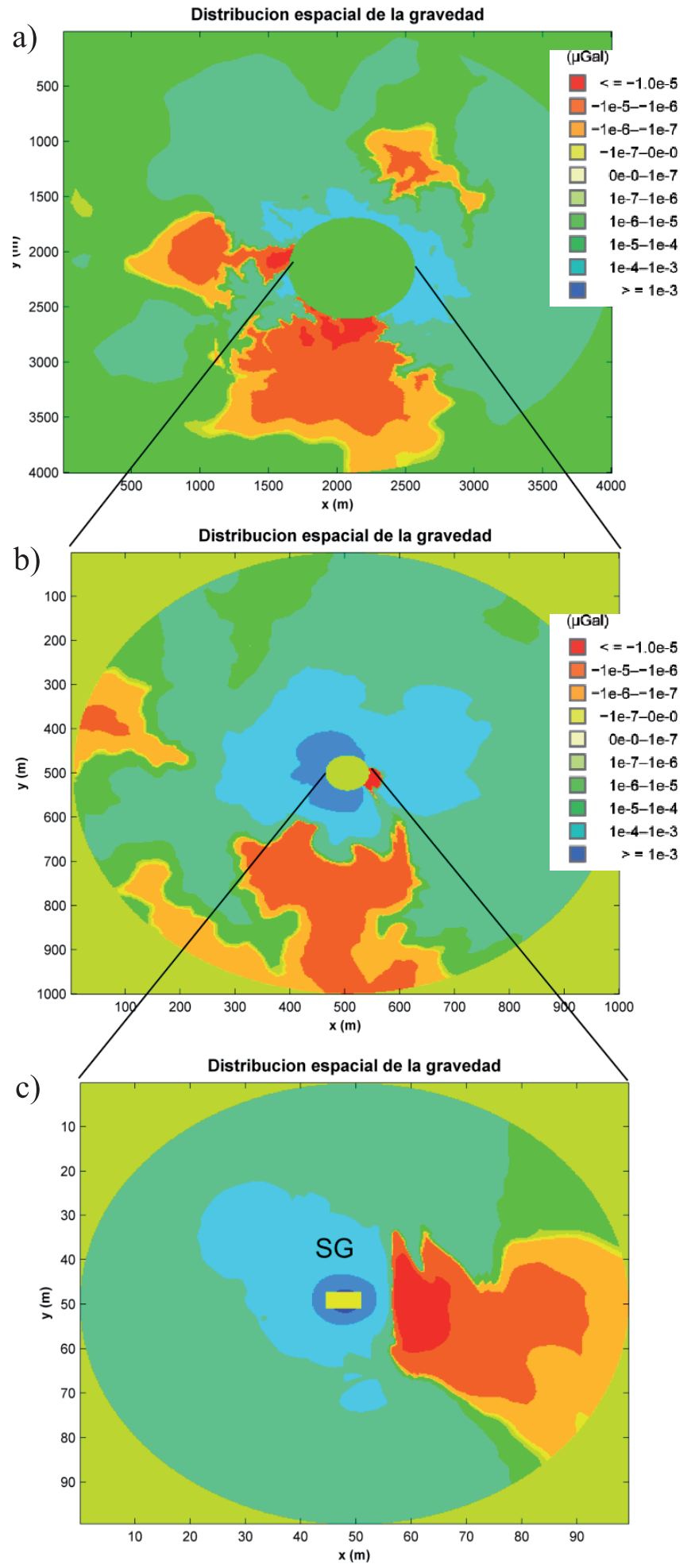

Figura 6: Distribución espacial de la variacion gravimétrica, para los diferentes radios, R: a) $2000 \mathrm{~m}$, b) $500 \mathrm{~m} \mathrm{y} \mathrm{c)} 50 \mathrm{~m}$.
Considerando las condiciones reales determinadas por la humedad medida en cada sensor, se obtiene la respuesta gravimétrica en el período desde enero 2006 hasta enero 2011, para una profundidad de análisis de hasta $2.6 \mathrm{~m}$ (Figura 7). Los valores de la respuesta son mayores cuando aumenta la profundidad.

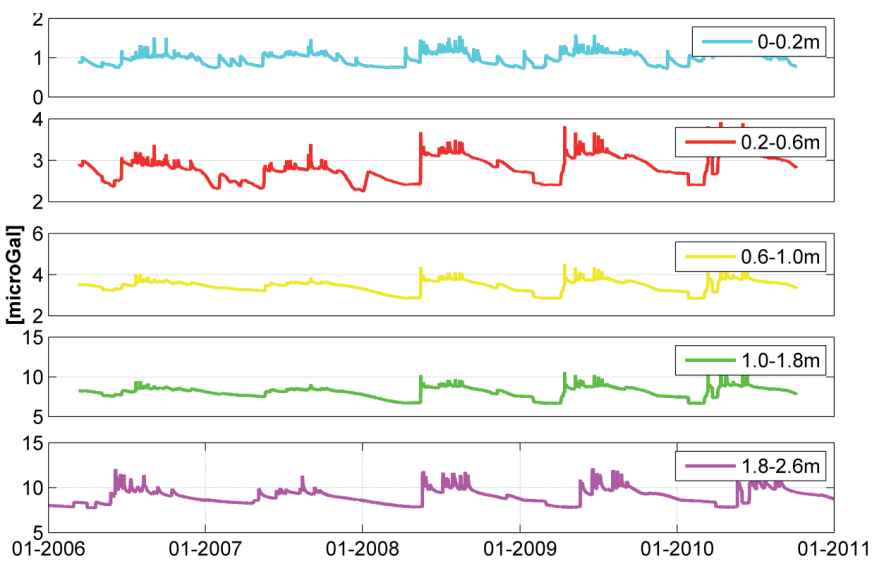

Figura 7: Respuesta gravimétrica de los diferentes horizontes

Sumando las respuestas gravimétricas de cada horizonte, se obtiene la respuesta total de la variación de la humedad en el suelo, ver Figura 8.

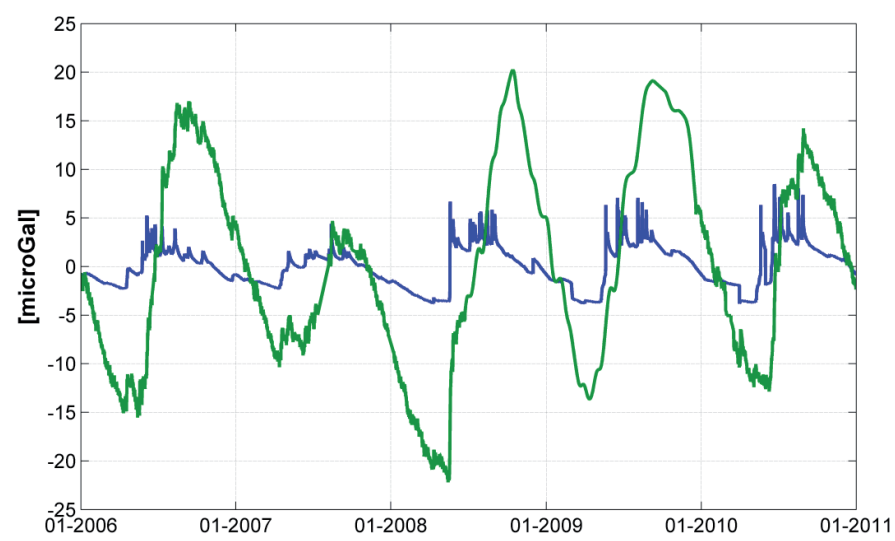

Figura 8: Respuesta gravimétrica hasta $2.6 \mathrm{~m}$ (línea azul) y gravedad residual SG38 (línea verde)

La respuesta gravimétrica de la humedad del suelo hasta los $2.6 \mathrm{~m}$ deja gran parte de la gravedad residual del SG sin ser explicada. Luego se aumenta la profundidad de análisis hasta $\operatorname{los} 20 \mathrm{~m}$ de profundidad considerando la humedad en la zona no saturada a los $2.2 \mathrm{~m}$ constante hasta $\operatorname{los} 20 \mathrm{~m}$, la respuesta gravimétrica aumenta (Figura 9), sin embargo, no explica el total de la respuesta. 


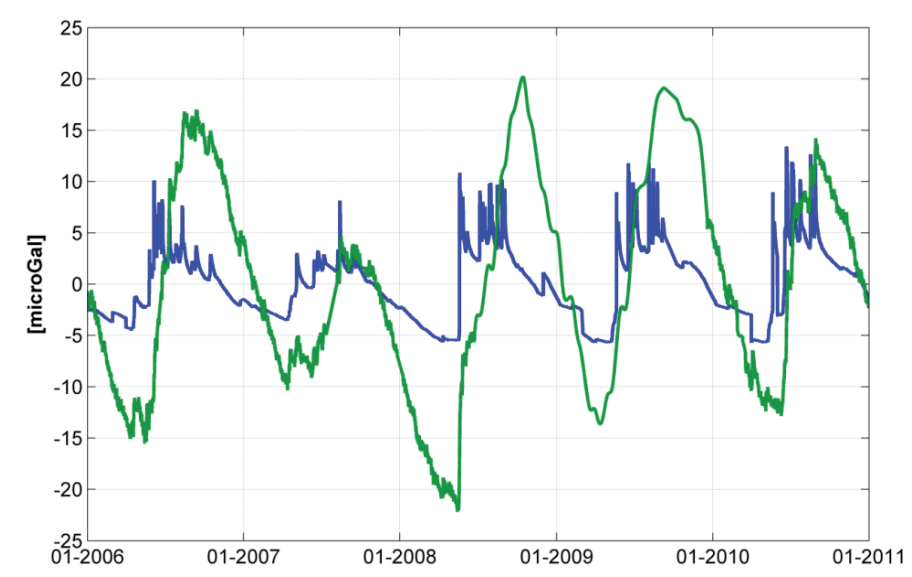

Figura 9: Respuesta gravimétrica hasta $20 \mathrm{~m}$ (línea azul) y gravedad residual SG38 (línea verde)

Para determinar el ajuste del modelo se realiza un análisis de regresión lineal del tipo mínima desviación absoluta (Least Absolute Deviation) considerando una profundidad de análisis de $2.6 \mathrm{~m}$ (Tabla 3), y luego considerando una profundidad de hasta $20 \mathrm{~m}$ (Tabla 4). Luego se calcula la correlación por rangos entre las variables para ambas profundidades (Tabla 5).

Tabla 3: Coeficiente de regresión de un modelo de regresión lineal simple, para una profundidad de hasta $2.6 \mathrm{~m}$

\begin{tabular}{|c|c|c|c|}
\hline Variable & Coeficiente & Desv. típica & $\mathrm{p}^{*}<$ \\
\hline Constante & 0.20 & 0.04 & 0.001 \\
\hline $\begin{array}{c}\text { Resp.gravimétrica } \\
2.6 \mathrm{~m}\end{array}$ & 3.88 & 0.02 & 0.001 \\
\hline
\end{tabular}

* nivel de significancia

Tabla 4: Coeficientes de regresión de un modelo de regresión lineal simple, para una profundidad de hasta $20 \mathrm{~m}$

\begin{tabular}{|c|c|c|c|}
\hline Variable & Coeficiente & Desv. típica & $\mathrm{p}^{*}<$ \\
\hline Constante & 0.43 & 0.04 & 0.001 \\
\hline $\begin{array}{c}\text { Resp. gravimétrica } \\
20 \mathrm{~m}\end{array}$ & 2.47 & 0.02 & 0.001 \\
\hline
\end{tabular}

* nivel de significancia

Tabla 5: Coeficiente de rango de correlación que existe entre la medición entregada por el SG38 y la respuesta de gravimétrica de la humedad del suelo.

\begin{tabular}{|c|c|c|}
\hline Profundidad, $\mathrm{m}$ & Coef. Correlación & $\mathrm{p}^{*}<$ \\
\hline 2.6 & 0.66 & 0.001 \\
\hline 20 & 0.67 & 0.001 \\
\hline
\end{tabular}

* nivel de significancia

Al determinar el error absoluto medio de cada modelo de regresión lineal, se puede observar en la Tabla 6 que el modelo, considerando una profundidad de análisis de 20 $\mathrm{m}$, mejora muy poco el ajuste.

Tabla 6: Error medio absoluto (MAE) de los modelos de regresión lineal

\begin{tabular}{|c|c|}
\hline Modelo & MAE $(\mu \mathrm{Gal})$ \\
\hline Respuesta gravimétrica $2.6 \mathrm{~m}$ & 3.80 \\
\hline Respuesta gravimétrica $20 \mathrm{~m}$ & 3.40 \\
\hline
\end{tabular}

La gráfica de la curva de gravedad residual es más suave que la gráfica de la respuesta gravimétrica al observar las Figuras 8 y 9, lo cual es otro indicador. La gran parte de la señal del SG que queda sin ser explicada a partir del cálculo de la respuesta gravimétrica, indicaría la falta de componentes por incluir. Sin embargo, la respuesta gravimétrica obtenida del modelo y la gravedad residual del SG muestran similar variación estacional, debido a las condiciones climáticas locales, las variaciones estacionales de la respuesta gravimétrica son de hasta $12 \mu \mathrm{Gal}$, para 2.6 $\mathrm{m}$ de profundidad.

\section{Conclusiones}

Al comparar los resultados de la respuesta gravimétrica con las mediciones gravimétricas entregadas por el gravímetro superconductor SG38, aunque presentan similitud en la variación estacional, podemos ver que existe una gran diferencia. Al aumentar la profundidad hasta los $20 \mathrm{~m}$, considerando la humedad del sensor ubicado a $2.2 \mathrm{~m}$, el resultado mejora levemente lo cual puede ser un indicador de que la diferencia entre la respuesta gravimétrica de la humedad del suelo y las mediciones del gravímetro superconductor SG38 se puede deber a variaciones dinámicas en la humedad del suelo o a la existencia de otros componentes involucrados que no fueron considerados. Por lo tanto, sería recomendable hacer mediciones y obtener las variaciones del contenido de agua en el suelo a profundidades mayores a $\operatorname{los} 2.6 \mathrm{~m}$, incluyendo variaciones de la napa freática.

Las curvas de los gráficos de gravedad residual provenientes del SG son mucho más suaves respecto a las curvas de los gráficos de respuesta gravimétrica, lo cual podría implicar una sobreestimación de la humedad en el modelo. Sin embargo, se sebe considerar que la señal 
gravimétrica del SG es una señal integrada que considera todos los almacenamientos, por lo tanto la falta de ajuste que presenta el modelo puede estar asociada a factores que no fueron considerados o a almacenamientos de humedad a mayor profundidad.

Son muchos los factores involucrados para obtener una respuesta precisa de las variaciones de humedad en el suelo, errores en las mediciones, hipótesis no precisas, variaciones espaciales que afectan la obtención de la respuesta gravimétrica, por lo que resulta difícil corregir las mediciones del gravímetro superconductor en base a las variaciones de humedad del suelo. Sin embargo, el uso de las mediciones de gravedad puede resultar de gran beneficio para la hidrología, ya que puede entregar información sobre los almacenamientos de agua, considerando que la señal del gravímetro es una señal integrada que considera todos los almacenamientos, es por ello que se debe ser muy cuidadoso, tanto en el manejo de los datos hidrológicos como en el manejo de los datos gravimétricos.

\section{Referencias}

Creutzfeldt, B., A. Güntner, T. Klügel, and H. Wziontek (2008). Simulating the influence of water storage changes on the superconducting gravimeter of the Geodetic Observatory Wettzell, Germany. Geophysics 73(6), WA95.
Creutzfeldt, B., Güntner, A., Thoss, H., Merz, B. and Wziontek, H. (2010). Measuring the effect of local water storage changes on in-situ gravity observations: Case study of the Geodetic Observatory Wettzell, Germany. Water Resources Research 46, W08531.

Davis, R.O.E. and Bennett, H.H. (1927). Grouping of soils on the basis of mechanical analysis. United States Departament of Agriculture. Departmental circulation No. 419.

Hasan, S. (2009). Terrestrial water storage change from temporal gravity variation Ph.D. thesis, Wagneingen University, Netherlands.

Hasan, S., Troch, P. A., Boll, J., and Kroner,C. (2006). Modeling the hydrological effect in local gravity at Moxa, Germany. Journal of Hydrometeorology 7(3), 346-354.

Leirião, S. (2007). Hydrological model calibration using ground based and spaceborne time-lapse gravity surveys. MSc thesis, Technical University of Denmark.

Tigo (2011): Observatorio Geodésico Integrado Transportable. Recuperado. Universidad de Concepción, octubre del 2011 de TIGO: http://www.tigo.cl/ 